$\xi=-$

\title{
The influence of longitudinal diffusion on the transport of dust particles emitted from a fixed source
}

\author{
Khaled S. Al-Mashrafi * \\ Section of Applied Sciences, Department of Human Resources Development, General Directorate \\ of Education in Sharqiyah South Governorate, Ministry of Education, Sur, Oman \\ *Corresponding author E-mail: khaled2014om@gmail.com
}

\begin{abstract}
The mathematical model for the diffusion of dust particles emitted from a fixed source in the presence of the longitudinal diffusion and absence of latitudinal and vertical diffusions, is investigated. The diffusion of dust particles in the atmosphere is governed by the atmospheric diffusion equation. In the previous paper [1], the general case of the time-dependent diffusion equation in the presence of a point source whose strength is dependent on time, was solved. The calculations showed that the diffusion parameters play an important role in the spread of the dust particles in the atmosphere. In the previous paper, we examined the model in the presence of vertical diffusion and absence of other diffusions to show that for small times, the dust spreads with a front that travels with the speed of the wind. In the current paper, the vertical and latitudinal diffusions are absent while the longitudinal diffusion is present. It is found that the solution depends on the source of time dependence. To study the nature of the solution, two special cases of the source are specified. In the both cases, it is found that there is no discontinuity front, and the dust particles spread slowly into the direction of the wind.
\end{abstract}

Keywords: Dust Diffusion; Atmospheric Diffusion Equation; Longitudinal Diffusion; Wind Speed; Unsteady State.

\section{Introduction}

The study of the transport of dust particles by wind in the atmosphere is relevant to some industrial [1] - [5] and environmental [6] - [10] applications. The existence of moderate to strong speed of wind can release particles of dust into the air, some of which can cause problems for humans, plants, and animals $[4 ; 6 ; 10]$. The study is also relevant to fume emitted by factories into the surroundings [5].

The spread of dust particles emitted from a certain source in the atmosphere is governed by the time-dependent atmospheric diffusion equation [11] - [16]:

$$
\frac{\partial \mathbf{C}}{\partial \mathrm{t}^{*}}+\mathbf{u} \cdot \nabla \mathbf{C}=\nabla \cdot(\underline{\mathbf{D}}: \nabla \mathrm{C})-\mathbf{w} \cdot \nabla \mathrm{C} ; \quad \nabla \cdot \mathbf{u}=0,
$$

in which $\mathrm{C}$ is the concentration of the dust particles, $\mathrm{t}^{*}$ is the time, $\mathbf{u}$ is the velocity vector, $\mathbf{w}$ is the settling velocity vector, and $\underline{\mathbf{D}}$ is the stress tensor.

The settling velocity, $\mathbf{w}$, depends mainly on the size and roughness of the particle [14].Since the gravity is the main effect on size, the settling velocity can be assumed to be vertical. In the current study, We assume that the dust particles are very light; hence the settling velocity can be ignored, so

$\mathbf{w}=\mathbf{0}$.

The wind speed has a logarithmic profile [16]. For areas near the ground, a linear dependence is a good approximation. In previous theoretical studies, wind speed was either constant, linear, or has a power-law in height $[8 ; 15]$. In this study, we shall assume that the flow is a non-zero constant. The diffusion tensor, $\underline{\mathbf{D}}$ is the local viscosity resulted from the friction force between dust particles in the atmosphere [1]. This force plays an important role in reducing the energy of the fluid and hence affecting the motion. In general, $\underline{\text { D }}$ is a function of position [8]. Here it is calculated by the theory of turbulence to be linear in distance in the direction of wind, when the wind speed is low [12]. Theoretical justification for this assumption has been provided by Taylor's statistical theory [17]. We consider that the diffusion tensor has the form.

$$
\underline{\mathbf{D}}=\left(\begin{array}{ccc}
\mathrm{D}_{\mathrm{x}^{*} \mathrm{x}^{*}} & 0 & 0 \\
0 & \mathrm{D}_{\mathrm{y}^{*} \mathrm{y}^{*}} & 0 \\
0 & 0 & \mathrm{D}_{\mathrm{z}^{*} \mathrm{z}^{*}}
\end{array}\right) \text {, }
$$

in a Cartesian system of coordinates in which $z^{*}-$ axis is vertically upwards, $x^{*}$-axis and $y^{*}$-axis are horizontal and the $x^{*}-$ axis is along the horizontal wind speed.

The transport of small dust particles (the diameter $<0.1 \mathrm{~mm}$ ) and its spread in the atmosphere has been studied theoretically by using the diffusion equation (1)[1]; [8 - 9]; [10 - 15]. The study by Al-Mashrafi [1] investigated the solution of the time-dependent diffusion equation in the presence of a point source whose strength is dependent on time and for low speeds $(<10 \mathrm{~m} / \mathrm{s})$. This model posed an initial boundary value problem for a second-order linear partial differential equation. The steady-state case of the same problem was studied by Sharan et al. [12] when the uniform source is situated at the ground level. In the both studies, the diffusion components were in three coordinate directions and were linearly proportional to the distance of wind direction. It was con- 
cluded that the height of the source above ground level plays an important role in the distribution of the pollutant in the atmosphere. Moreover, it was shown that the diffusion parameters play an essential role in the spread of dust particles emitted from a fixed source in the atmosphere. In the previous study [1], we assumed that the diffusion in the vertical direction is potent, while the diffusions in the both horizontal directions (longitudinal and latitudinal diffusions) are absent. It was found that for small values of the time, the particles of dust spared with a front that travels with the wind speed.

In the present study, we intend to investigate the solution when the longitudinal diffusion is potent and the latitudinal and the vertical diffusions are absent. The mathematical model is derived in section 2. The atmospheric diffusion equation depends on the horizontal distance, $x$ and the time, $t$. In section 3, the time-dependent diffusion equation with the relevant initial and boundary conditions is solved, and the solution is obtained in the domain $(x, t)$ for a general function of source. In section 4 , two special cases of the source are taken to study the dependence of the longitudinal diffusion on the solution. The solutions are presented in figures. In section 5, some conclusions are summarized.

\section{Formulation of the model}

The diffusion of dust particles emitted from a certain source situated on/or above ground level in the atmosphere is governed by the atmospheric diffusion equation (1). This equation can be written in a Cartesian system of coordinates $\mathrm{O}\left(\mathrm{x}^{*}, \mathrm{y}^{*}, \mathrm{z}^{*}\right)$ in the absence of the settling velocity as

$$
\begin{aligned}
& \frac{\partial \mathrm{C}}{\partial \mathrm{t}^{*}}+\mathbf{u} \cdot \nabla \mathrm{C}=\frac{\partial}{\partial \mathrm{x}^{*}}\left(\mathrm{D}_{\mathrm{x}^{*} \mathrm{x}} \frac{\partial \mathrm{C}}{\partial \mathrm{x}^{*}}\right) \\
& +\frac{\partial}{\partial \mathrm{y}^{*}}\left(\mathrm{D}_{\mathrm{y}^{*} \mathrm{y}^{*}} \frac{\partial \mathrm{C}}{\partial \mathrm{y}^{*}}\right)+\frac{\partial}{\partial \mathrm{z}^{*}}\left(\mathrm{D}_{\mathrm{z}^{*}} \mathrm{z}^{*} \frac{\partial \mathrm{C}}{\partial \mathrm{z}^{*}}\right),
\end{aligned}
$$

where $\mathrm{Oz}^{*}$ is vertically upwards, $\mathrm{Ox}^{*}$ and $\mathrm{Oy}^{*}$ are horizontal, $\mathrm{C}\left(\mathrm{x}^{*}, \mathrm{y}^{*}, \mathrm{z}^{*}, \mathrm{t}^{*}\right)$ is the concentration of the dust particles in the time $\mathrm{t}^{*}, \mathbf{u}$ is the velocity of dust particles, and $\left(D_{\mathrm{x}^{*} x^{*}}, D_{y^{*} y^{*}}, D_{z^{*} z^{*}}\right)$ are the components of the stress tensor in $\mathrm{x}^{*}, \mathrm{y}^{*}, \mathrm{z}^{*}$ directions respectively. Equation (4) is basically equation of continuity that conserves the amount of dust particles in the atmosphere. It has two sides: the rate of change of dust on the lefthand side, and the flux on the right-hand side.

Consider the $x^{*}$-axis is oriented in the direction of the wind speed, and the velocity of the dust particles is given by

$$
\mathbf{u}=(\mathrm{U}, 0,0)+(\mathrm{u}, \mathrm{v}, \mathrm{w}),
$$

where $(\mathrm{U}, 0,0)$ is the wind speed, and $(\mathrm{u}, \mathrm{v}, \mathrm{w})$ is velocity of dust particles relative to the local wind speed. The components $\mathrm{u}, \mathrm{v}, \mathrm{w}$ are very small in comparison with $\mathrm{U}$, so that.

$$
|(\mathrm{u}, \mathrm{v}, \mathrm{w})| \ll \mathrm{U} .
$$

By using the equations (5) - (6), the advection term in (4) reduces to

$$
\mathbf{u} \cdot \nabla \mathrm{C} \sim \mathrm{U} \frac{\partial \mathrm{C}}{\partial \mathrm{x}^{*}}+\mathrm{v} \frac{\partial \mathrm{C}}{\partial \mathrm{y}^{*}}+\mathrm{w} \frac{\partial \mathrm{C}}{\partial \mathrm{z}^{*}} .
$$

$\mathbf{u} . \nabla \mathrm{C} \sim \mathrm{U} \frac{\partial \mathrm{C}}{\partial \mathrm{x}^{*}}$.

In the case of moderate to high wind speeds, diffusion components are proportional to the vertical height. For low wind speeds, diffusion components depend on the distance downwind. We assume that the components of the diffusion tensor are proportional to the horizontal direction $\mathrm{x}^{*}$, so

$$
\begin{array}{cc}
D_{\mathrm{X}^{*} \mathrm{x}^{*}}=\alpha \mathrm{Ux}^{*}, & \mathrm{D}_{\mathrm{y}^{*} \mathrm{y}^{*}}=\beta \mathrm{Ux}^{*}, \\
\mathrm{D}_{\mathrm{Z}^{*} \mathrm{z}^{*}}=\gamma \mathrm{Ux}^{*} ; & \alpha, \beta, \gamma \geq 0 .
\end{array}
$$

Then substituting (8) - (9) into equation (4) to get

$$
\begin{aligned}
& \frac{\partial \mathrm{C}}{\partial \mathrm{t}^{*}}+\mathrm{U} \frac{\partial \mathrm{C}}{\partial \mathrm{x}^{*}}=\alpha \mathrm{U} \frac{\partial}{\partial \mathrm{x}^{*}}\left(\mathrm{x}^{*} \frac{\partial \mathrm{C}}{\partial \mathrm{x}^{*}}\right) \\
& +\beta \mathrm{U} \frac{\partial}{\partial \mathrm{y}^{*}}\left(\mathrm{x}^{*} \frac{\partial \mathrm{C}}{\partial \mathrm{y}^{*}}\right)+\gamma \mathrm{U} \frac{\partial}{\partial \mathrm{z}^{*}}\left(\mathrm{x}^{*} \frac{\partial \mathrm{C}}{\partial \mathrm{z}^{*}}\right) .
\end{aligned}
$$

The relevant initial and boundary conditions then are:

(i) Far away from the point source, the concentration decreases to zero:

$$
\mathrm{C}\left(\mathrm{x}^{*}, \mathrm{y}^{*}, \mathrm{z}^{*}, \mathrm{t}^{*}\right) \rightarrow 0 \quad \text { as } \quad \mathrm{x}^{*},\left|\mathrm{y}^{*}\right|, \mathrm{z}^{*} \rightarrow \infty,
$$

(ii) The source at $\mathrm{z}^{*}=\mathrm{h}^{*}$ is switched on at a certain time $\mathrm{t}^{*}=0$, so the concentration vanishes for $\mathrm{t}^{*} \leq 0$ :

$\mathrm{C}\left(\mathrm{x}^{*}, \mathrm{y}^{*}, \mathrm{z}^{*}, 0\right)=0$

(iii) The source at $\left(0,0, \mathrm{~h}^{*}\right)$ has a strength $\overline{\mathrm{Q}}$ for all $\mathrm{t}^{*}>0$ :

$$
\begin{aligned}
& \mathrm{C}\left(0, \mathrm{y}^{*}, \mathrm{z}^{*}, \mathrm{t}^{*}\right)=\frac{\overline{\mathrm{Q}}}{\mathrm{U}} \delta\left(\mathrm{y}^{*}\right) \delta\left(\mathrm{z}^{*}-\mathrm{h}^{*}\right) \mathrm{f}\left(\mathrm{t}^{*}\right) \\
& \text { with } \mathrm{f}(0)=0 ; \mathrm{t}^{*}>0,
\end{aligned}
$$

(iv) The flux of the dust particles at $\mathrm{z}^{*}=0$ vanishes:

$$
\frac{\partial \mathrm{C}\left(\mathrm{x}^{*}, \mathrm{y}^{*}, 0, \mathrm{t}^{*}\right)}{\partial \mathrm{z}^{*}}=0
$$

in which the function $\delta(\mathrm{s})$ is Dirac's delta function. The condition (14) is acceptable because the level $\mathrm{z}^{*}=0$ is impermeable and particles cannot penetrable it.

The equation (10) subject to the conditions (11) - (14) was solved in the study by Al-Mashrafi [1]. It was found that the concentration in the $\left(\mathrm{x}^{*}, \mathrm{y}^{*}, \mathrm{z}^{*}\right)$ plane at all times $\mathrm{t}^{*}$ depends on the diffusion parameters, the wind speed, the source, and the position of the particles in the atmosphere. To understand the effect of the diffusion parameters on the spread of the dust particles, we investigated the case $\{\alpha=\beta=0, \gamma \neq 0\}$ in the previous study [1]. It was found that the extent of transport of dust depends on the strength of the source. When the source is strong for small times, the solution showed a discontinuity. 
3. The solution in the case of longitudinal diffusion $\{\beta=\gamma=0, \alpha \neq 0\}$

In the present study, we assume the diffusion along the $\mathrm{x}$ - direction is present but diffusions in $\mathrm{z}-$ and $\mathrm{y}-$ directions are absent, so that $D_{y y}=D_{z z}=0$. In this case, the concentration is independent of $\mathrm{z}$ and $\mathrm{y}$, so the relevant atmospheric diffusion equation is given by

$$
\frac{\partial \mathbf{C}}{\partial \mathrm{t}^{*}}+\mathrm{U} \frac{\partial \mathrm{C}}{\partial \mathrm{x}^{*}}=\alpha \mathrm{U} \frac{\partial}{\partial \mathrm{x}^{*}}\left(\mathrm{x}^{*} \frac{\partial \mathrm{C}}{\partial \mathrm{x}^{*}}\right)
$$

subject to the initial and boundary conditions:

$$
\mathrm{C}\left(\mathrm{x}^{*}, 0\right)=0 \text {, }
$$

$\mathrm{C}\left(\mathrm{x}^{*}, \mathrm{t}^{*}\right) \rightarrow 0 \quad$ as $\mathrm{x}^{*} \rightarrow \infty$,

$\mathrm{C}\left(0, \mathrm{t}^{*}\right)=\frac{\overline{\mathrm{Q}}}{\mathrm{U}} \mathrm{f}\left(\mathrm{t}^{*}\right) ; \mathrm{f}(0)=0$.

We note here that the source is situated at $\mathrm{x}=0$ and its height does not affect the problem. In reality, this represents a fixed infinite line source at $\mathrm{x}=0$.

The equation (15) and the conditions (16) - (18) can be reduced to

$$
\begin{aligned}
& \frac{\partial \mathrm{C}}{\partial \mathrm{t}}+\frac{\partial \mathrm{C}}{\partial \mathrm{x}}=\alpha \frac{\partial}{\partial \mathrm{x}}\left(\mathrm{x} \frac{\partial \mathrm{C}}{\partial \mathrm{x}}\right), \\
& \mathrm{C}(\mathrm{x}, 0)=0, \\
& \mathrm{C}(\mathrm{x}, \mathrm{t}) \rightarrow 0 \quad \text { as } \mathrm{x} \rightarrow \infty, \\
& \mathrm{C}(0, \mathrm{t})=\mathrm{Qf}(\mathrm{t}) ; \mathrm{f}(0)=0,
\end{aligned}
$$

where we have defined

$$
\mathrm{t}=\mathrm{Ut}^{*}, \quad \mathrm{x}=\mathrm{x}^{*}, \quad \mathrm{Q}=\frac{\overline{\mathrm{Q}}}{\mathrm{U}} .
$$

The equation (19) relevant to the conditions (20) - (22) can be solved straightforward by using the integral transforms method to get the solution

$$
\mathrm{C}(\mathrm{x}, \mathrm{t})=\frac{\mathrm{Q}}{\Gamma(2 \mu)} \int_{0}^{\mathrm{t}} \mathrm{g}(\mathrm{t}-\mathrm{s}) \mathrm{s}^{2 \mu-1} \mathrm{e}^{-2 \mu \mathrm{x} / \mathrm{s}} \mathrm{ds},
$$

in which $\Gamma(\mathrm{r})$ is the Gamma function of argument $\mathrm{r}$ and $\mathrm{g}(\mathrm{t})$ is the inverse Laplace transform of $F(\omega)$ with respect to the time $t$ given by

$$
\mathrm{g}(\mathrm{t})=\mathrm{L}^{-1}\left\{\mathrm{~F}(\omega) \omega^{2 \mu}\right\},
$$

, $F(\omega)$ is the Laplace transform of the function $f(t)$ with respect to $\mathrm{t}$ defined by

$$
F(\omega)=\int_{0}^{\infty} f(t) e^{-\omega t} d t,
$$

and we have assumed the notation

$$
\eta=2 \sqrt{2 \mu \omega}, \quad \mu=\frac{1}{2 \alpha} .
$$

Expression (24) represents the general solution for the concentration of dust particles subject to diffusion present only in the direction of the wind in closed form. The behavior of the solution depends on the source $f(t)$ as represented by $g(t)$ in (24). This expression specifies the concentration at every point $(x, t)$ of the domain. This solution, with specific forms of $f(t)$, will be discussed in detail in the next section.

\section{Discussion}

The expression (24) gives the solution for a general function $\mathrm{f}(\mathrm{t})$ when longitudinal diffusion $\left(\mathrm{D}_{\mathrm{xx}}\right)$ is present, and both vertical and latitudinal diffusions $\mathrm{D}_{\mathrm{zz}}$ and $\mathrm{D}_{\mathrm{yy}}$ respectively, are absent. This model does not depend on height $\mathrm{z}$, or lateral distance $\mathrm{y}$. It then represents a source fixed along the $z$-axis at $(0,0,0)$ and having an infinite length $(z \geq 0)$. We will study here the nature of the solution of this case by using two examples of the function $f(t)$. Let us take the two examples of function $f(t)$ which are: (i) Heaviside function $\mathrm{H}(\mathrm{t})$ and (ii) exponential function $1-\mathrm{e}^{-\lambda \mathrm{t}}, \lambda>0[1]$

\subsection{The source function with $\mathbf{f}(t)=H(t)$}

The solution (24) in this case is given by the expression

$$
\begin{aligned}
& \mathrm{C}(\mathrm{x}, \mathrm{t})=\frac{\mathrm{Q}}{\Gamma(2 \mu)}(\mathrm{q})^{\mu-\frac{1}{2}} \mathrm{e}^{\frac{-\mathrm{q}}{2}} \mathrm{~W}_{\mu-\frac{1}{2}, \mu}(\mathrm{q}) ; \\
& \mathrm{q}=\frac{2 \mu \mathrm{x}}{\mathrm{t}},(2 \mu<1, \quad \alpha>1),
\end{aligned}
$$

where $\mathrm{W}_{\mathrm{a}, \mathrm{b}}(\mathrm{x})$ is the Whittaker function of the second kind with argument $\mathrm{x}$ and parameters $\mathrm{a}$ and $\mathrm{b}$ [18 - 19].

Expression (28) represents the solution for the concentration of dust particles in the case of a source that is uniform with time. The Whittaker function of the second kind can be written in the form of the Confluent hypergeometric function of the second kind $\mathrm{U}(\mathrm{a}, \mathrm{b}, \mathrm{x})[18]$, hence the expression (28) reduces to

$\mathrm{C}(\mathrm{x}, \mathrm{t})=\frac{\mathrm{Q}}{\Gamma(2 \mu)}(\mathrm{q})^{2 \mu} \mathrm{e}^{-\mathrm{q}} \mathrm{U}(1,2 \mu+1, \mathrm{q}) ; \mu<\frac{1}{2}$.

It is informative to consider the limiting cases of the solution (29) with the time $t$ and distance $x$. When $q \rightarrow \infty$, i.e. $\left(t \rightarrow 0^{+}, x \neq 0\right)$, the Confluent hypergeometric function reduces to [19]

$$
\mathrm{U}(\mathrm{a}, \mathrm{b}, \mathrm{q}) \sim \mathrm{q}^{-\mathrm{a}}\left[\sum_{\mathrm{n}=0}^{\mathrm{R}-1} \frac{(\mathrm{a})_{\mathrm{n}}(1+\mathrm{a}-\mathrm{b})_{\mathrm{n}}}{\mathrm{n} !}(-\mathrm{q})^{-\mathrm{n}}\right]+\mathrm{O}(|\mathrm{q}|)^{-\mathrm{R}},
$$

where, $\mathrm{R}^{\text {th }}$ term is the smallest of the terms in the series expansions of $U(a, b, q)$, and

$$
(a)_{n}=a(a-1)(a-2) \ldots \ldots(a-n+1) \quad, \quad(a)_{0}=1 .
$$

Then

$$
\mathrm{U}(1,2 \mu+1, \mathrm{q}) \sim \mathrm{q}^{-1}\left[\begin{array}{c}
1+\frac{1-2 \mu}{\mathrm{q}}+ \\
\frac{2(1-2 \mu) 2 \mu}{2 \mathrm{q}^{2}}+\ldots \ldots . .
\end{array}\right]=\mathrm{q}^{-1}\left\{1+\mathrm{O}\left(\frac{1}{\mathrm{q}}\right)\right\} .
$$




$$
\mathrm{C}(\mathrm{x}, \mathrm{t}) \sim \frac{\mathrm{Q}(\mathrm{q})^{2 \mu-1} \mathrm{e}^{-\mathrm{q}}}{\Gamma(2 \mu)}\left\{1+\mathrm{O}\left(\frac{1}{\mathrm{q}}\right)\right\} \quad ; \quad \mu<\frac{1}{2},
$$

which can be simplified to

$$
\mathrm{C}(\mathrm{x}, \mathrm{t}) \rightarrow 0 \quad \text { as } \mathrm{q} \rightarrow \infty(\text { i.e. } \mathrm{t} \rightarrow 0, \mathrm{x} \neq 0) \text {. }
$$

Similarly, when $\mathrm{q} \rightarrow 0$ (i.e. $\mathrm{t} \rightarrow \infty$ ),

$$
\mathrm{U}(\mathrm{a}, \mathrm{b}, \mathrm{q}) \sim \frac{\Gamma(\mathrm{b}-1)}{\Gamma(\mathrm{a})} \mathrm{q}^{1-\mathrm{b}}+\mathrm{O}(1), \quad 1<\mathrm{b}<2 .
$$

Then,

$$
\mathrm{U}(1,2 \mu+1, \mathrm{q}) \sim \Gamma(2 \mu) \mathrm{q}^{-2 \mu}+\mathrm{O}(1)
$$

and (29) reduces to

$$
\mathrm{C}(\mathrm{x}, \mathrm{t}) \rightarrow \mathrm{Q} \quad \text { as } \mathrm{t} \rightarrow \infty .
$$

The solution (28) can be written in terms of the Incomplete Gamma function $\gamma(\mathrm{a}, \mathrm{x})$ [19] by using the relation

$$
\gamma(\mathrm{a}, \mathrm{x})=\Gamma(\mathrm{a})-\mathrm{x}^{\left(\frac{\mathrm{a}-1}{2}\right)} \mathrm{e}^{\frac{-\mathrm{x}}{2}} \mathrm{~W}_{\frac{\mathrm{a}-1}{2}, \frac{\mathrm{a}}{2}}(\mathrm{x}),
$$

where $\gamma(\mathrm{a}, \mathrm{x})$ is defined by

$$
\gamma(\mathrm{a}, \mathrm{x})=\int_{0}^{\mathrm{x}} \mathrm{e}^{-\mathrm{t}} \mathrm{t}^{\mathrm{a}-1} \mathrm{dt} \quad ; \mathrm{a}>0
$$

Hence solution (28) reduces to

$$
\mathrm{C}(\mathrm{x}, \mathrm{t})=\frac{\mathrm{Q}}{\Gamma(2 \mu)}\left\{\Gamma(2 \mu)-\gamma\left(2 \mu, \frac{2 \mu \mathrm{x}}{\mathrm{t}}\right)\right\}
$$

Using (27) to write the solution (40) in the case of diffusion in the wind direction as

$$
\mathrm{C}(\mathrm{x}, \mathrm{t})=\frac{\mathrm{Q}}{\Gamma\left(\frac{1}{\alpha}\right)}\left\{\Gamma\left(\frac{1}{\alpha}\right)-\gamma\left(\frac{1}{\alpha}, \frac{\mathrm{x}}{\alpha \mathrm{t}}\right)\right\} \quad ; \alpha>1 \text {. }
$$

For the cases, $x=0$ and $x \rightarrow \infty$, we use (39) and note that [18]

$$
\gamma(2 \mu, 0)=0 ; \quad \gamma(2 \mu, \infty)=\Gamma(2 \mu)
$$

It then follows that

$$
\begin{aligned}
& \mathrm{C}(0, \mathrm{t})=\frac{\mathrm{Q}}{\Gamma(2 \mu)}\{\Gamma(2 \mu)-\gamma(2 \mu, 0)\}=\mathrm{Q}, \\
& \mathrm{C}(\mathrm{x}, \mathrm{t}) \rightarrow \frac{\mathrm{Q}}{\Gamma(2 \mu)}\{\Gamma(2 \mu)-\gamma(2 \mu, \infty)\} \rightarrow 0 \quad ; \text { as } \mathrm{x} \rightarrow \infty .
\end{aligned}
$$

The asymptotic values (34), (37), (43) - (44) confirm the conditions $(20)-(22)$ for $C(x, t)$ of this case.

Another special case occurs when $\alpha=2.0$ when the solution (41) reduces to a simpler form. In such case, the Incomplete Gamma function can be written as $\gamma\left(\frac{1}{2}, \frac{x}{2 t}\right)$, and then using the following relation [19]

$$
\operatorname{erfc}(y)=1-\frac{1}{\sqrt{\pi}} \gamma\left(\frac{1}{2}, y^{2}\right)
$$

where erfc(y) is the Complementary Error function defined by

$\operatorname{erfc}(y)=\frac{2}{\sqrt{\pi}} \int_{y}^{\infty} e^{-t^{2}} d t$

Then the solution (41) becomes

$C(x, t)=Q \operatorname{erfc}\left(\sqrt{\frac{x}{2 t}}\right) ; \quad \alpha=2.0$

The contours of the solution (41) in the $(x, t)$ plane are illustrated for different values of the diffusion parameter $\alpha$ in figure 1 . The Incomplete Gamma function in (41) was computed using a standard NAG library subroutine by using its definition in (39). As a check on these results we have used the case $\alpha=2.0$ where a simpler solution in (47) is obtained. The contours of the solution (47) are presented in figure 2 .

From figures 1 and 2, we can see that at every point of the space in the $(x, t)$ plane the intensity of dust particles decreases whenever the diffusion parameter $\alpha$ increases. For a fixed value of the distance $\mathrm{x}$, the concentration of dust increases with time $\mathrm{t}$. That means at every point at a distance $\mathrm{x}$ from the source, the concentration will increase as the time increases. This is expected; the concentration will increase as the source releases more particles of dust. Moreover, at a specific time $t$, the concentration of dust decreases when we move far away from the source. This last situation can happen in real life because when we move away from the source, the dust will be so far from the source and there is not enough force to carry the particles that far. The figures also show that the particles diffuse in the horizontal direction as well as with time.

The values of $\alpha$ chosen for figure 1 are made so that comparison between the solutions (41) and (47) is made easy. If we compare figure 1(b) with figure 2 for $\alpha=2.0$, we see that the computations for both cases are consistent. 

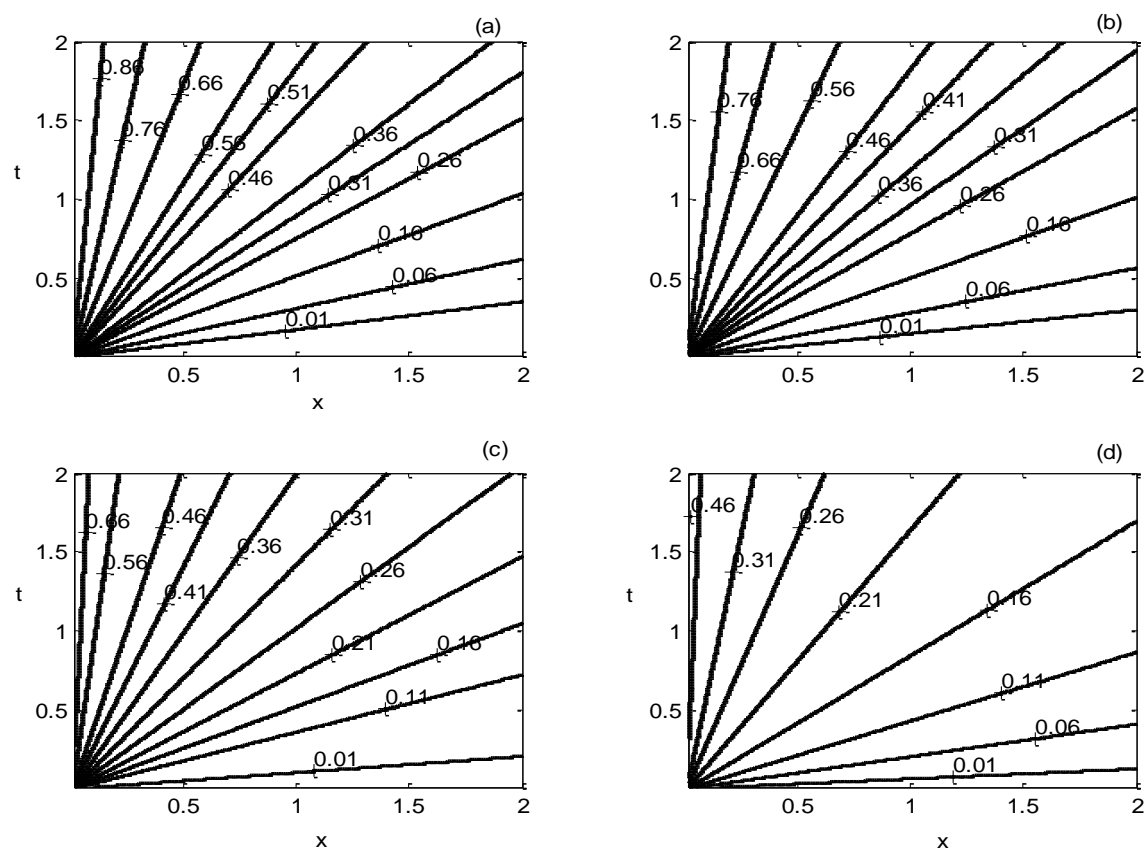

Fig. 1: The isolines of the concentration $\bar{C}(x, t)=C(x, t) / Q$ in the $(x, t)$ plane for the function $f(t)=H(t)$ for some values of the diffusion parameter: (a) $\alpha=1.5$, (b) $\alpha=2.0$, (c) $\alpha=4.0$, and (d) $\alpha=10.0$. Note the increasing of the longitudinal diffusion parameter.

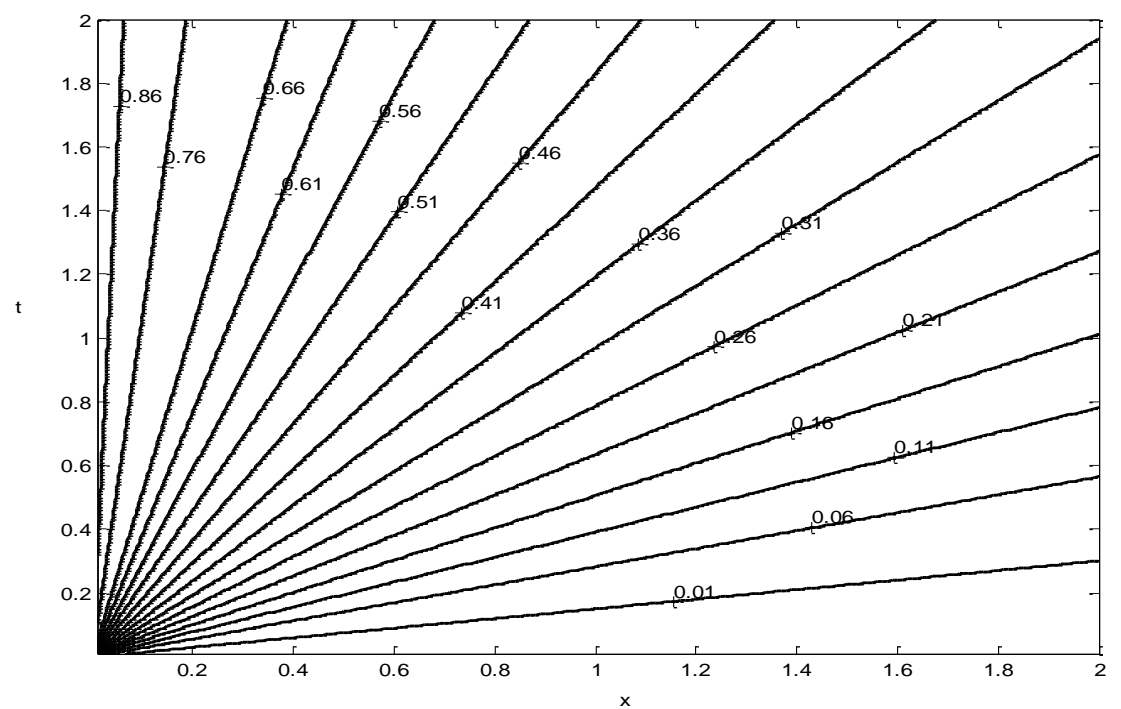

Fig. 2: The isolines of the concentration $\bar{C}(x, t)=C(x, t) / Q$ in the $(x, t)$ plane for the function $f(t)=H(t)$ for a special case of complementary error function when $\alpha=2.0$, in the case when longitudinal diffusion only is present. Compare this with figure 1(b).

\subsection{The source function with $f(t)=1-e^{-\lambda t}, \lambda>0$}

Using the expression (24) to find the solution for this case as

$$
\mathrm{C}(\mathrm{x}, \mathrm{t})=\frac{\mathrm{Q} \lambda}{\Gamma(2 \mu) \Gamma(2-2 \mu)} \int_{0}^{\mathrm{t}}\left(\frac{\mathrm{t}}{\mathrm{s}}-1\right)^{1-2 \mu} \mathrm{e}^{\frac{-2 \mu \mathrm{x}}{\mathrm{s}}} \mathrm{M}(1,2-2 \mu
$$$$
, \lambda(s-t)) d s,
$$

where $\mathrm{M}(\mathrm{a}, \mathrm{b}, \mathrm{c})$ is the Confluent hypergeometric function of the first kind [19].

The integrand in (48) appears to have a singularity at $s=0$. To study the limit of this expression as $\mathrm{s} \rightarrow 0$, assume

$$
A=\lim _{y \rightarrow \infty}\left\{y^{1-2 \mu} e^{-2 y \mu x}\right\} ; \quad y=\frac{1}{s}
$$

Direct substitution gives, $(\infty / \infty)$. However, using L'Hopital's rule gives

$$
A=\lim _{y \rightarrow \infty}\left\{\frac{(1-2 \mu) y^{-2 \mu}}{(2 \mu x) e^{2 \mu x y}}\right\}=0 ; \quad \text { since } 0<\mu<\frac{1}{2} .
$$

Hence, the limit of the expression $(s)^{2 \mu-1} e^{\frac{-2 \mu x}{s}}$ at $s=0$ is finite, so the integration is finite and the conclusion is that the solution (48) exits.

Figures 3 and 4 below show the contours of the concentration (48) in the $(x, t)$ plane for different values of the decay factor $\lambda$ with two specific values of the coefficients of the longitudinal diffusion; $\alpha=2.0$ and $\alpha=10.0$, respectively. The intensity of dust particles at every point of the $(x, t)$ plane increases whenever the 
decay factor $\lambda$ increases for a fixed value of longitudinal diffusion $\alpha$. This situation must happen in real life because when the coefficient of the decay increases, the strength of the source increases. Hence, the source produces more particles.

When the values of $\alpha$ and $\lambda$ are fixed, then the concentration of dust particles increases as the time increases for a fixed distance $\mathrm{x}$. Furthermore, at a specific time $\mathrm{t}$ for fixed strength of the source and longitudinal diffusion, the concentration decreases when we move far away from the source.

It can be deduced from figures 3 and 4 that for small values of $\lambda$ and fixed $\alpha$, the diffusion of the dust particles in the horizontal direction is very weak, and the dust needs more time to diffuse in the $x$ - direction, (see figures $3(a, b)$, and $4(a, b)$ ). As $\lambda$ increases, the diffusion steadily increases. For large values of the decay factor $\lambda$, the dust approaches the steady-state solution, (see figure $3(\mathrm{c}, \mathrm{d}))$. The large values of $\lambda$ resulted in the strong source and whenever these values increase further the strength of the source converges to the steady state (figure $4(\mathrm{c}, \mathrm{d})$ ).

For a fixed value of the decay factor $\lambda$ at every point in the plane, the concentration when $\alpha=2.0$ is more than that when $\alpha=10.0$, (see figure 3 (a, b, c, d) and compare it with figure $4(a, b, c, d)$, respectively).

Comparison between the two cases of the function $f(t)$ in the presence of longitudinal diffusion shows that the force of the source in case (4.1) is stronger than that for the source in case (4.2). But the two solutions will be identical for large values of the decay factor $\lambda$, for $t>0$, when the strengths of the two sources become equal. The spread of the dust in the horizontal direction in case (4.2) is weaker than that for case (4.1) for $t>0$. This difference resulted from the difference in the strength of the source.
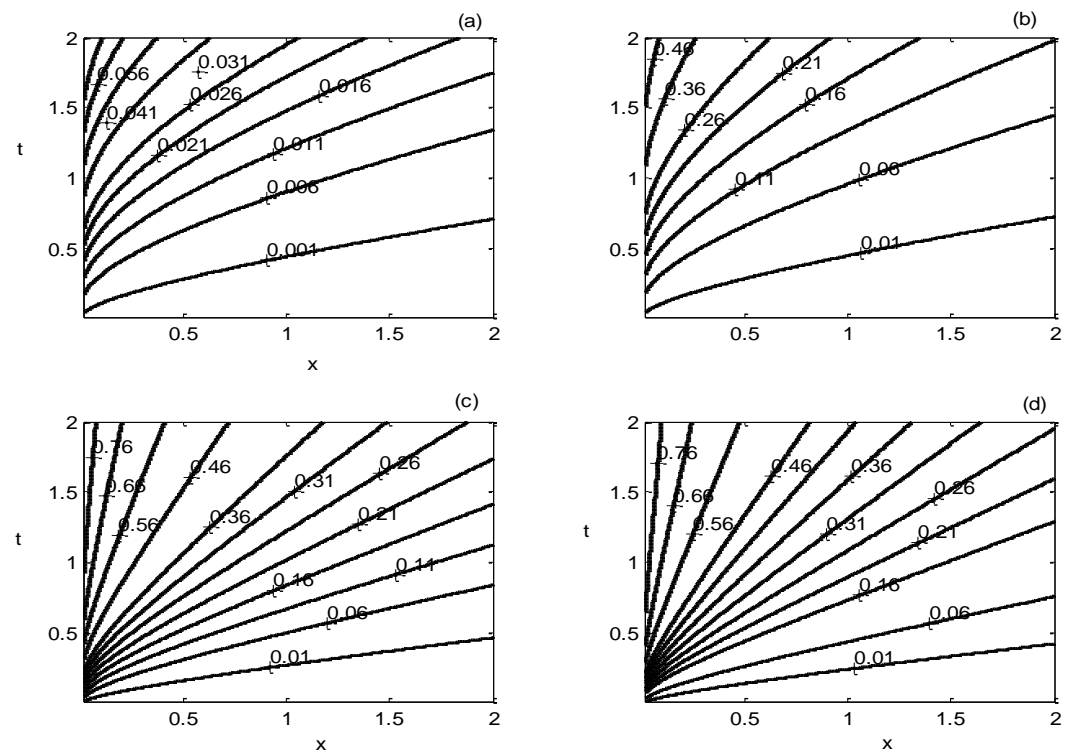

Fig. 3: The profile of the concentration $\bar{C}(x, t)=C(x, t) / Q$ for a fixed value of $\mu=0.25(\alpha=2.0)$ and different values of the decay factor: (a) $\lambda=0.1$, (b) $\lambda=1.0$, (c) $\lambda=6.0$, and (d) $\lambda=10.0$. Compare with figure 1(b) for general case of $\alpha$ and with figure 2 for special case of error function solution for $\alpha=2.0$.
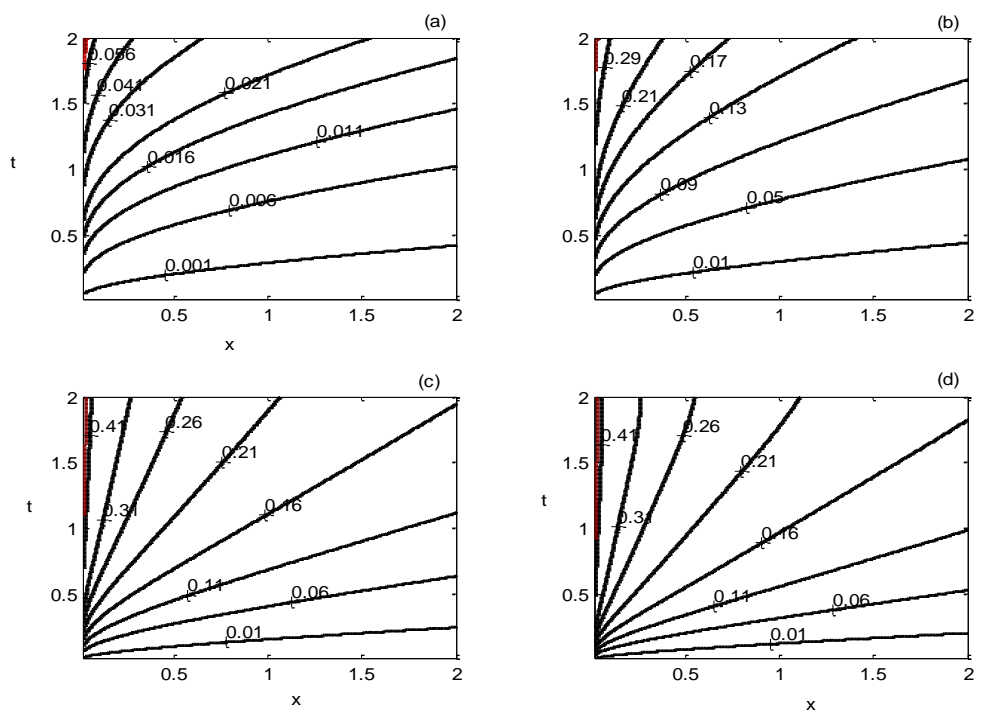

Fig. 4: The profile of the concentration $\overline{\mathrm{C}}(\mathrm{x}, \mathrm{t})$ for a fixed value of $\mu=0.05(\alpha=10.0)$ and different values of decay factor: (a) $\lambda=0.1$, (b) $\lambda=1.0$, (c) $\lambda=5.0$, and (d) $\lambda=10.0$. Note the increasing coefficient of decay, and compare that with figure 1 (d) when $\alpha=10.0$.

It can be observed from figures 1 - 4 above that the solution of the case (4.2) with specific value of $\alpha$ approaches the solution of case (4.1) with same value of $\alpha$ when the decay coefficient $\lambda$ increases rapidly. This observation can be proved analytically when the factor of decay $\lambda$ goes to $\infty$ by recalling the asymptotic form of the Confluent hypergeometric function of the first kind [18] and then substituting it into the expression (48) to find that this expression is identical with the expression (28). 


\section{Conclusion}

A mathematical model for the diffusion of dust particles emitted from a fixed source in the presence of longitudinal diffusion and absence of vertical and latitudinal diffusions has been studied. The atmospheric diffusion equation in this case depends on the horizontal distance, $\mathrm{x}$, and time, $\mathrm{t}$. An analytical expression of the concentration of dust particles for a general source function, $\mathrm{f}(\mathrm{t})$ at every point of the domain $(x, t)$ is obtained in closed form. Two special functions of the source have been tested to investigate the nature of the solution and its dependence on the source. The solutions are presented graphically in figures $1-4$. The profiles of the solutions showed that the concentration of dust particles in the $(x, t)$ plane depends on the parameter of diffusion in the direction of the wind. The strong presence of this parameter led to the distribution of the dust in a larger area. In comparison with the previous study [1] when longitudinal and latitudinal diffusions where absent and vertical diffusion was present, it is concluded here that there is no discontinuity front and the particles of dust spread slowly into the direction of the wind.

\section{References}

[1] Al-Mashrafi, K.S., "Diffusion of dust particles emitted from a fixed source", International Journal of Applied Mathematical Research, 4 (4), (2015), pp: 454-465. http://dx.doi.org/10.14419/ijamr.v4i4.5058.

[2] Roberts, O.F.T., "The theoretical scattering of smoke in a turbulent atmosphere", Proc. Roy. Soc. London Ser. A, 104, (1923), pp: 640654. http://dx.doi.org/10.1098/rspa.1923.0132.

[3] Llewelyn, R.P., "An analytical model for transport, dispersion and elimination of air pollutants emitted from a point source", Atmospheric Environment, 17, (1983), pp: 249-256. http://dx.doi.org/10.1016/0004-6981(83)90040-9.

[4] Skidmore, E.L., "Soil erosion by wind: an overview, in physics of desertification" (F. EL-Baz and M. H. Hassan, eds.), Martinus Nijhoff Publishers, Dordrecht, (1986), pp: 261-273.

[5] Kevin B.M., Howard R.B. and Ronald G.R., "Numerical simulation of smoke plumes from large oil fires", Atmospheric Environment, 30, (1996), pp: 4125-4136. http://dx.doi.org/10.1016/13522310(96)00151-3.

[6] Bagnold, R.A., "The physics of Blown sand and Desert Dunes", Methuen, London, (1954).

[7] Kennedy, J.F., "The formation of sediment ripples, dunes and antidunes", Ann. Rev. Fluid Mech., 1, (1969), pp: 147-168. http://dx.doi.org/10.1146/annurev.fl.01.010169.001051.

[8] Eltayeb, I.A. and Hassan, M.H.A., "Two-dimensional transport of dust from an infinite line source at ground level", Geophys. J. Int., .110, (1992), pp: 571-576. http://dx.doi.org/10.1111/j.1365246X.1992.tb02092.x.

[9] Eltayeb, I.A. and Hassan, M.H.A., "The Evolution of dust emitted by a uniform source above ground level", International Journal of Mathematics and Mathematical Sciences, (2003), pp: 3327-3343. http://dx.doi.org/10.1155/S0161171203106163.

[10] Gillette, D.A., "Threshold Friction Velocities for Dust production for Agricultural Soils”. J. Geophys. Res., 93, (1988), pp: $12645-$ 12662. http://dx.doi.org/10.1029/JD093iD10p12645.

[11] Gillette, D. and Goodwin, P., "Microscale transport of sand-sized soil aggregates eroded by wind", J. Geophys. Res, 79, (1974), pp: 4080-4084. http://dx.doi.org/10.1029/JC079i027p04080.

[12] Sharan M., Singh M.P., and Yadav, A.K., "Mathematical model for atmospheric dispersion in low winds with eddy diffusivities as linear functions of downwind distance", Atmospheric Environment, 30 (1996), pp: 1137-1145. http://dx.doi.org/10.1016/13522310(95)00368-1.

[13] Sharan M., and Gupta S., "Two-dimensional analytical model for estimating crosswind-integrated concentration in a capping inversion: eddy diffusivity as a function of downwind distance from the source", Atmospheric Environment, 36, (2002), pp: 97-105. http://dx.doi.org/10.1016/S1352-2310(01)00449-6.

[14] Eltayeb, I.A. and Hassan, M.H.A., "Diffusion of dust particles from a point source above ground level and a line source at ground level", Geophys. J. Int., 142, (2000), pp: 426-438. http://dx.doi.org/10.1046/j.1365-246x.2000.00172.x.
[15] Hassan, M.H.A. and Eltayeb, I.A., "Suspension transport of wind eroded sand particles", Geophys. J. Internet. , (1991b), 104, pp: 147-152. http://dx.doi.org/10.1111/j.1365-246X.1991.tb02500.x.

[16] Pasquill, F., "Atmospheric Diffusion", D. Van Nostrand, Princeton, (1962).

[17] Taylor G.I., "Diffusion by continuous movements". Proc. London Math. Soc., 20, (1921), pp: 196-212.

[18] Zwillinger, D., "Handbook of Differential Equations", Mass, Academic Press, Boston, (1989).

[19] Abramowitz, M. and Stegun, I., "Handbook of Mathematical Functions, with Formulas, Graphs and Mathematical Tables", Dover, New York, (1965). 